\title{
Inorganic Nanoparticles for Cancer Treatment
}

\author{
Created by: Heidi Abrahamse \\ Hanieh Montaseri , Cherie Kruger
}

Version received: 18 May 2020

check for

updates

The application of porphyrins and their derivatives have been investigated extensively over the past years for phototherapy cancer treatment. Phototherapeutic Porphyrins have the ability to generate high levels of reactive oxygen with a low dark toxicity and these properties have made them robust photosensitizing agents. In recent years, Porphyrins have been combined with various nanomaterials in order to improve their bio-distribution. These combinations allow for nanoparticles to enhance photodynamic therapy (PDT) cancer treatment and adding additional nanotheranostics (photothermal therapy-PTT) as well as enhance photodiagnosis (PDD) to the reaction. This review examines various porphyrin-based inorganic nanoparticles developed for phototherapy nanotheranostic cancer treatment over the last three years (2017 to 2020). Furthermore, current challenges in the development and future perspectives of porphyrin-based nanomedicines for cancer treatment are also highlighted.

\section{Cancer Phototherapies with Porphyrin PS-Based Inorganic Nanoparticles}

The advent of NPs has allowed for the majority of conventional PS limitations to be overcome. NPs are small in size and range between 1 to $100 \mathrm{~nm}$ in diameter and some can contain/carry multiple theranostic agents [1]. Furthermore, their large surface to volume ratios can enhance the payload of PS delivered to target cells [2]. NPs can also prevent premature release of PS, which consequently can prevent nonspecific accumulation in healthy cells [3]. Importantly, NPs give amphilicity to PSs in order to bypass through the blood stream unaffected [4]. Since NPs are small in size when conjugated to PSs, they enhance passive diffusion into tumor cells through the EPR effect [5] [6]. Furthermore, PS-NP conjugates can be bound to various targeting ligands to improve active targeted delivery of PSs into deep seated tumors, resulting in a high accumulation of the therapeutic agent and improved PDD or PDT outcomes [5].

Drug delivery systems based on inorganic NPs provide the advantages of a wide surface area conjugation chemistry with versatile surface functionalization [7]. In addition, they bestow great biocompatibility, easy preparation, and excellent physico-chemical properties in order to enhance therapeutic efficacy [7]. It is important to appreciate that nonbiodegradable nanoparticles, including inorganic NPs, have recently drawn considerable research interest in the field of PDT, as multifunctional theranostic carriers, due to their unique optical properties and size, shape, and porosity tunability, however is out of the scope of this review. Furthermore, when organic NPs are compared with inorganic polymeric biodegradable NPs, they do not degrade easily into biological systems and do not release a PS efficiently after activation, instead they allow oxygen species to seep out of the carrier. Thus, inorganic NPs (which is the focus of this review) are considered far more superior as they can carry many PSs to the tumor cells as a single nanoplatform, with far more beneficial outcomes [1].

Porphyrins such as protoporphyrin IX (PpIX), purpurin, and benzoporphyrins are the second-generation PSs ${ }^{[8][9][10][11] . ~}$ These types of porphyrins are important PS agents in cancer phototherapy due to their structural presence of $\mathrm{N}$ - $\mathrm{H}$ groups and nitrogen atoms, whereby they can be further functionalized by having either nanoparticles, metal ion chelates or polymers conjugated onto their surfaces [12][13]. Since first- and second-generation porphyrins have a high ability to produce ROS in phototherapy cancer applications, they are lipophilic in nature [14], have a low dark toxicity and low water solubility, as well as bio-distribution and their overall emission quantum yields are greatly affected [8][15]. Furthermore, the low passive selectivity of porphyrins for tumor tissue can cause remarkable off-target and unwanted normal tissue damage [16]. Thus, more studies are now focusing on porphyrin-NP conjugated systems for higher phototherapy efficiency, in relation to concentrated tumor cell uptake and so overall improved ROS production for PDT and PTT cancer treatments [1].

\section{Porphyrin-Based Noble Metallic Nanoparticles}

Noble metallic NPs such as gold, silver, and platinum have unique properties such as ease of functionalization due to 
their different chemical moieties and broad optical properties [17]. Additionally, noble metallic NPs can be fine-tuned to desired wavelengths required for porphyrin phototherapies to be effective [17][18]. Furthermore, the localized surface plasmon resonance (LSPR) of gold nanoparticles (AuNPs) ${ }^{[19]}$ and Au metallic components allow for photothermal conversion in cancer phototherapeutic treatments and so contribute to the overall effectivity of the treatment via tumor theranostic heat destruction [20]. However, the low biocompatibility of noble metal NPs sometimes causes restrains within in vivo applications [5].

Within studies performed by Penon et al. (2017), AuNPs conjugates were synthesized using biphasic and monophasic methods to investigate the optimal method of synthesis [21]. These synthesized AuNPs were then conjugated to a porphyrin PS derivative containing an alkanethiol (5-[4-(11-mercaptoundecyloxy) phenyl]-10,15,20-triphenylporphyrin, PR-SH) and a thiolated carboxyl terminated polyethylene glycol (PEG) counterpart [21]. The results from these studies reported that the monophasic method of AuNPs produced the highest amount of singlet oxygen, and so was utilized for PDT treatment assays on in vitro cultured SK-BR-3 human breast cancer [21]. Additionally, the porphyrin-based monophasic AuNPs were also covalently functionalized with a specific breast cancer targeting antibody (Ab) antierbB2, to enhance cellular uptake [21]. Overall, a higher cellular uptake was achieved when using the porphyrin-based monophasic-AuNP-PEG-Ab conjugate and a more significant cellular membrane damage was attained after PDT irradiation, when compared to controls [21].

Metalloporphyrins which contain Zinc (Zn) also provide a high PDT efficiency when compared to porphyrins alone, due to their metallic photothermal contribution [22]. However, due to their low solubility and distribution, they are often found conjugated to various vehicles like NPs in order to overcome these shortfalls [23]. Within studies by Alea-Reyeset al. (2017) AuNPs were synthesized with thiol-PEG to promote water solubility and stabilized with amphiphilic gemini-type pyridinium salt [23]. Onto these AuNPs an anionic molecule of $\mathrm{Zn}(\mathrm{II})$ meso-tetrakis(4-carboxyphenyl) porphyrin (ZnTCPP) was incorporated [23]. The synthesized Zn-TCPP loaded AuNPs generated remarkable amounts of singlet oxygen for the PDT treatment of in vitro human breast cancer cell line (SKBR-3), when compared to normal epithelium cells (MCF-10A) [23].

Over the past few years, researchers have shown that the combination of PDT and PTT has higher treatment efficiency than when compared to these treatments in singular form [니][25][26]. In a study, by Zhang et. al. (2019) they integrated synergistic PDT and PTT treatment of in vitro A549 cells and in vivo lung cancer mouse models, using 660 and $808 \mathrm{~nm}$ laser irradiations [27]. They fabricated 4-carboxyphenyl porphyrin conjugated silica-coated gold nanorods (AuNR@SiO ${ }_{2}$-TCPP), in which the AuNRs acted as the photothermal conversion agent for PTT, while the TCPP porphyrin PS produced ROS for effective PDT treatment [27]. This study reported that the coating of AuNRs with mesoporous silica, improved PS loading capability and overall drug biocompatibility. In vitro experiments post phototherapy treatment noted a significant cell death of $79 \%$ of cultured lung cancer cells [27]. Within in vivo AuNR@SiO 2 -TCPP phototherapy treated mouse models, a remarkable inhibition of tumor volumes was found when compared to control mouse model groups which did not receive treatment. In addition, treated mice with PDT/PTT and AuNR@SiO2-TCPP showed a significant reduction tumor volumes, while AuNRs/PTT and PDT/ AuNR@SiO $2-T C P P$ treatments alone produced minimal effect [27].

Since AuNPs exhibit a high tendency to aggregate, researchers have overcome this issue by coating them hydrophilic polymers such as chitosan polyethylene glycol (PEG) or hyaluronic acid in order to promote stability, as well as prevent reduction of heat conversion properties [28]. Additionally, these polymers not only enhance stability and solubility AuNPs, but also reduce their overall biotoxicity [29]. In this regard, studies performed by Zenget al. (2018) AuNPs were modified with chitosan (QCS-SH) via ligand exchange and then were attached to a PS porphyrin derivative, known as meso-tetrakis (4-sulphonatophenyl) porphyrin (TPPS) for dual PDT and PTT therapy (Figure 1) ${ }^{[16]}$. This nanohybrid (TPPS/QCS-SH/AuNPs) produced high singlet oxygen and noted high elevated temperature of $56^{\circ} \mathrm{C}$ applications when compared to AuNPs or TPPS treatment alone, suggesting that this drug has potential for applications in tumor phototherapy fields $[16]$. 


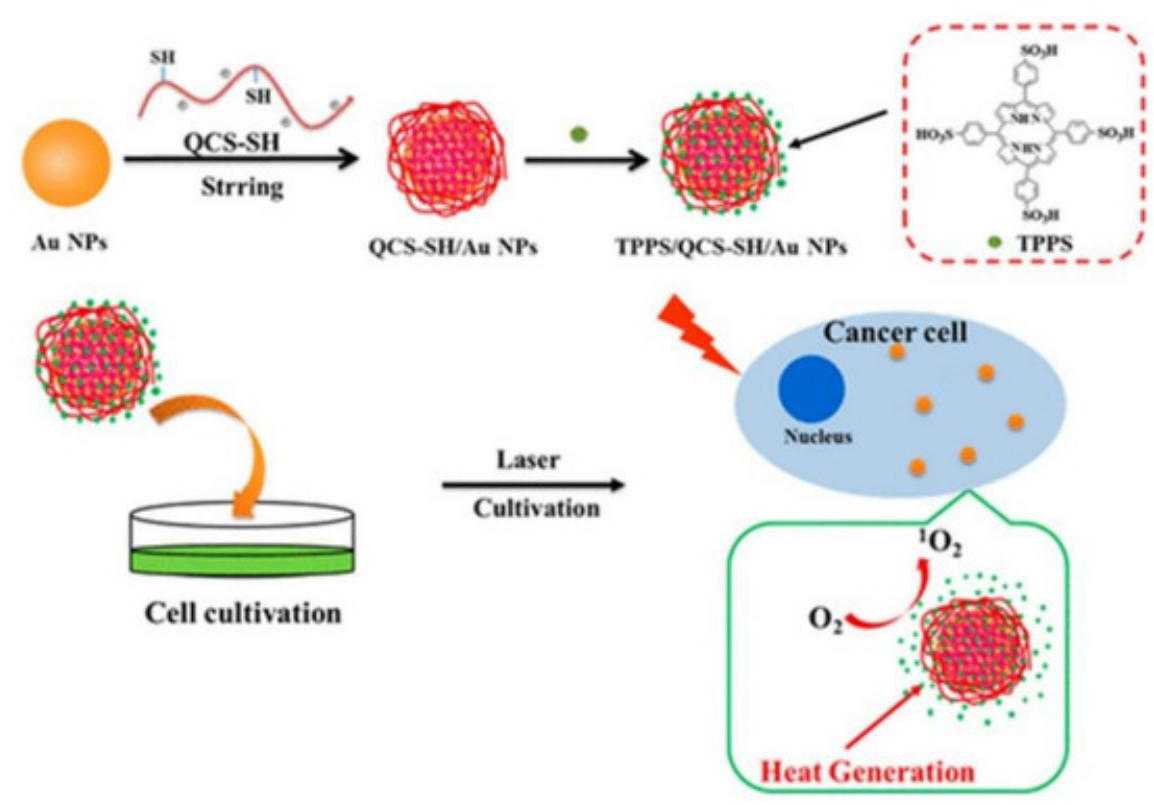

Figure 1. Proposed fabrication of meso-tetrakis (4-sulphonatophenyl) porphyrin (TPPS)/QCS-SH/gold nanoparticles (AuNPs) for dual mode photodynamic therapy (PDT)/photothermal therapy (PTT) treatment of cancer. Reprinted with permission from ref. ${ }^{[16]}$. Copyright 2018 American Chemical Society.

With respect to the combination of PDT and PTT to enhance synergistic phototherapeutic effects, Wei el al. (2018) employed cyclomatrix-type polyphosphazenes to prepare nanosized cross-linked polyphosphazene materials [30]. A tetra(4-hydroxyphenyl) porphyrin (TPP) PS with phenolic hydroxyl groups was then cross linked to this polymer material to form poly(cyclotriphosphazene-co-tetraphenylporphyrin-co-sulfonyldiphenol) nanospheres (CP-TPP) [30] . PEG-AuNPs were then conjugated onto the CP-TPP nanospheres for PDT $(630 \mathrm{~nm})$ and PTT $(808 \mathrm{~nm})$ in vitro treatment of cervical cancer HeLa cell lines [30]. The abundant presence of nitrogen and phosphorous atoms, as well as phenolic hydroxyl groups on the surface of polyphosphazene materials allow for its successful binding to the surface of AuNPs [31][32]. During characterization experiments the PEG-AuNPs-CP-TPP nanospheres demonstrated high surface plasmon resonance in the infrared (NIR) region for PDT applications and high localized temperature under laser irradiation for PTT assays ${ }^{[33]}$. Overall, the in vitro cytotoxicity results from this study showed that under sequential irradiation at $630 \mathrm{~nm}$ and $808 \mathrm{~nm}$, the cervical cancer cells viability decreased to $10 \%$, when the highest concentration of the drug conjugate was applied and so confirmed the effectiveness of combined PDT and PTT for enhanced cancer therapeutics $[30]$.

Nonselective activation or universal aggregation-caused quenching (ACQ) has greatly decreased the efficiency of conventional PSs for PDT clinical applications [34][35]. ACQ is the main setback in conventional PSs, since they have extended $\pi$-conjugation chains, when used at high concentrations and so their fluorescence is reduced PSs remarkably. When a PS aggregates, strong intermolecular $\pi-\pi$ stacking interactions occur leading to quenched emission via nonradiative pathways. Thus, conventional PSs can only be utilized at low concentrations, however this affects their photostability [36]. Furthermore, it has been reported that nonselective activation or nonspecific drug leakage of conventional PSs can occur during blood circulation or diffusion into normal tissues which exert unwanted therapy-related side effects, such as toxicity and drug resistance [35].

Furthermore, within clinical trials it had been noted that PDT patients sometimes become photosensitive and so are required to stay away from light to prevent unwanted PS activation until it is completely absorbed by tumor cells, in order to prevent unwanted damage to normal tissues [37]. In this sense, smart PSs have opened a growing research field of PSs. Smart PSs generally remain in the "Off" state during the absorption period and are only selectively activated or turned "On" once they have been fully internalized by cancer tumor cells [38]. Recently, a new phototherapy strategy based on NIR smart PS platforms was proposed by Huang and co-workers (2019) to evaluate its in vitro and in vivo PDT efficiency in breast cancer (4T1) cells and murine tumor induced models 39 . They integrated porphyrin PS units into upper critical solution temperature (UCST) block copolymer decorated gold nanorods (AuNR-P(AAm-co-AN-co-TPP)-b-PEG) [39]. The AuNRs acted as a NIR-manipulated PDT smart PS, as well as a fluorescence quencher of the porphyrin PS and photothermal producer [39]. Results noted that during blood 
circulation, the UCST block of the copolymer formed a collapsed-core and so caused aggregation of porphyrin PS units, subsequently leading to its "Off" state [39]. Upon internalization of the smart PS nanoplatform into cancer cells and NIR irradiation at $808 \mathrm{~nm}$, the $\pi-\pi$ stacking between the porphyrin units broke, activating the PS via a phase transition of UCST polymers from a collapsed to an extended state, causing the porphyrin PS unit to turn "On" (Figure 2) [39]. In this "On" state and localized state within tumor cells, the porphyrin PS then received $650 \mathrm{~nm}$ PDT laser irradiation to more effectively generate ROS and singlet oxygen, in order boost the phototherapy efficacy of this smart PDT treatment ${ }^{[39}$. Furthermore, the NIR irradiation allowed for a photothermal heating reaction to occur within the AuNRs, which were contained within this drug conjugate and so added to the overall phototherapy efficacy of this smart PDT PS treatment [39]. Overall, this smart PDT "Off/On" state process could be well manipulated using hybrid nanoplatforms with UCST block copolymers and AuNRs, and so could open new prospects for clinical-based PDT treatments ${ }^{[39]}$. Regarding these in vivo studies, although the tumor growth in the mice injected with the nanoplatforms under 808 and $650 \mathrm{~nm}$ laser were remarkably increased, the therapeutic effect was similar to the mice treated with the nanoplatform under $650 \mathrm{~nm}$ laser only [39].

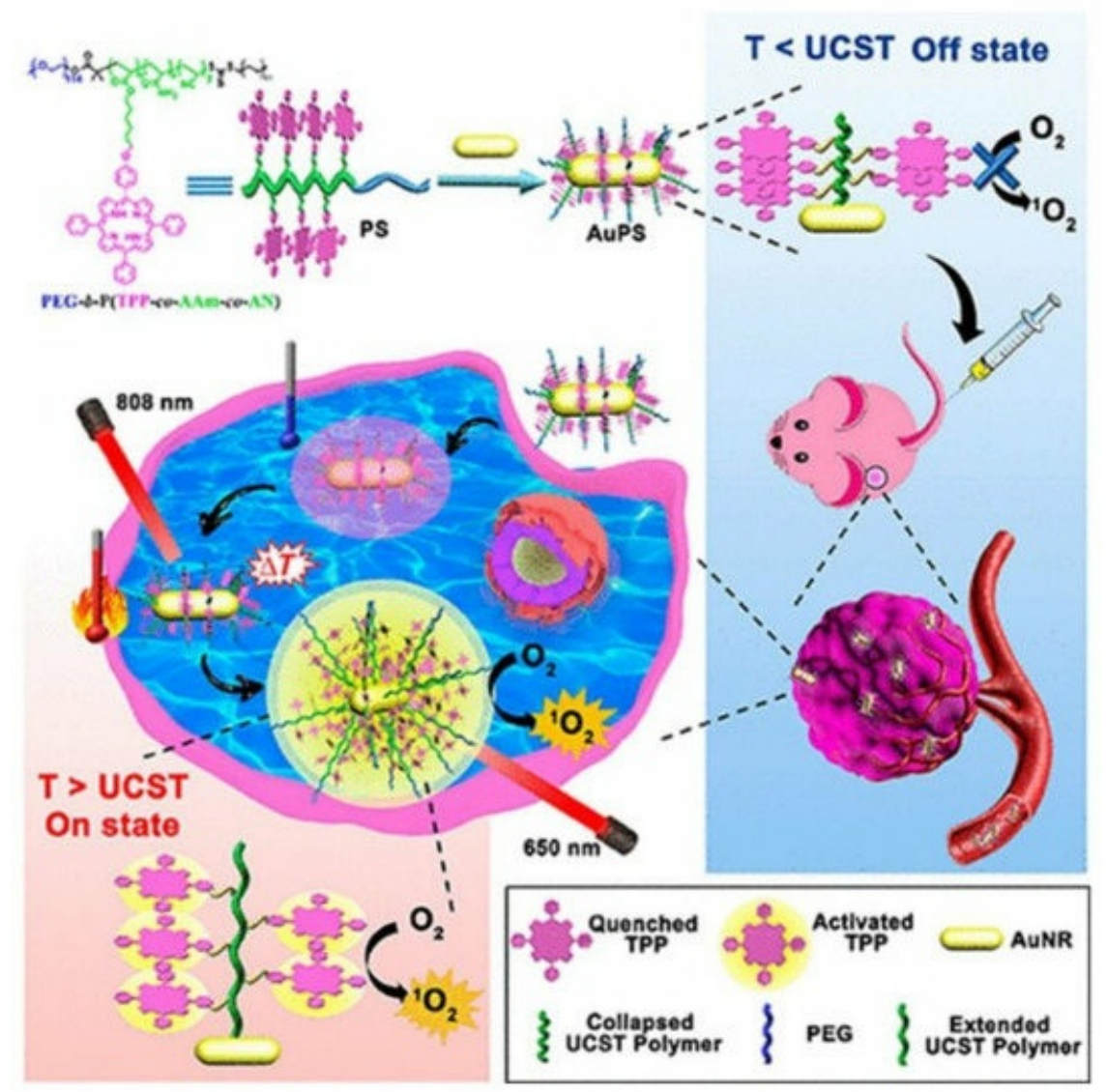

Figure 2. Schematic preparation of the "Off/On" upper critical solution temperature (UCST) smart nanoplatform process for the PDT clinical treatment in murine breast cancer models. Reprinted with permission from ref. [39]. Copyright 2019 American Chemical Society.

Generally, phototherapeutic agents are mainly locate in adjacent regions of a tumor, due to their abnormal vasculature nature and high interstitial fluid pressure (IFP), thus large parts of the tumor remain unaffected, since they do not adequately absorb PSs [40]. However, evidence has shown that many types of important cells associated with tumor initiation and progression are those fed by the defective blood vessels which supply solid tumors [41][42] and research must focus on phototherapy research to target these cells, which in turn can prevent tumor growth and metastases [43]. Various strategies have focused on adjacent cells, while those distant from blood vessels remain untouched [44]. Therefore, the development of novel strategies to improve the penetration and uptake properties of PSs still remains an obstacle in nanotherapeutics.

Two-photon PDT (2P-PDT) is a promising treatment approach that uses the absorption of two photons with low energy in NIR region (650-950 $\mathrm{nm}$ ) to provide deeper penetration, as well as a accurately target cancer tissues ${ }^{45}$. Some conventional PSs tend to have a week emission and lack of imaging possibilities and 2P-PDT can improve these limitations since this modality consists of small two-photon absorption (2PA) cross sections [45][46]. 
Inspired by 2P-PDT, Li et al. (2019) successfully conjugated poly [9,9'-bis(6"-bromohexyl) fluorene-2,7-ylenevinyleneco-alt-1,4-phenylene] (PFV) as a two-photon light harvesting material to tetraphenylporphyrin (TPP) to improve its PDT efficiency [47]. Conjugated polymer nanoparticles (CPNs) were then covalently attached to silica coated AuNRs (AuNR@SiO ${ }_{2}-\mathrm{CPNs}$ ) [47]. Fluorescence resonance energy transfer from PFV and plasmon resonance through AuNRs enhanced the two-photon properties of the TPP [47]. The nanohybrid was further applied for simultaneous two-photon imaging and 2P-PDT in vitro treatment of Hela cells which revealed significant enhancement in two-photon excitation fluorescence (980-fold) and two-photon induced singlet oxygen generation efficiency (792-fold) to eradicate cervical cancer cells $[47$.

A new treatment modality known as sonodynamic therapy (SDT) was proposed by Lianget al. (2019). SDT is used to excite and so activate PSs to produce high enough ROS levels for effective cancer therapy [48]. Furthermore, as already mentioned, photothermal absorbers or photothermal transduction agents (PTAs) are adopted in PTT to harvest light energy and generate hyperthermia ${ }^{449}$. These PTAs are categorized into semiconductor nanocrystals (NCs) ${ }^{[50]}$, inorganic materials [51], and organic dyes [52]. It has been reported that platinum or palladium-based photothermal transduction agents (PTAs) as inorganic PTAs have a higher photothermal stability and better catalytic properties than when compared to Au-based PTAs [53][54]. Furthermore, studies have noted that copper sulfide semiconductor NCs provide very high LSPR within the NIR region [55].

In this regard, studies developed platinum-copper sulfide Janus nanoparticles conjugated to tetra-(4-aminophenyl) porphyrin (TAPP) to overcome the low penetration depth of PDT [48]. In fact, they integrated semiconductor NCs and noble platinum metal to form their PTAs [48]. The noble platinum metal promoted the photothermal conversion efficiency under $808 \mathrm{~nm}$ laser irradiation by changing the electron transport pathway ${ }^{[56]}$ and the large space of the hollow copper sulfide NPs interior facilitated a high loading capacity of the TAPP PSs ${ }^{[48]}$. The synthesized nanohybrid was further coated with a temperature-sensitive polymer consisting of (poly (oligo (ethylene oxide) methacrylate-co-2(2-methoxyethoxy) ethyl methacrylate) to increase the biocompatibility and temperature triggered drug controlled release ${ }^{488}$. Therefore, upon laser irradiation and catalysis-enhanced SDT, this PS nanocarrier produced hyperthermia and a large amount of singlet oxygen which successfully managed to eradicate CT26 murine colon cancer cells within in vitro experiments with a cell viability of $19.8 \%$. Thein vivo studies using the CT26 xenograft tumor model in the group treated with the nanohybrid under laser and ultrasound irradiation demonstrated complete removal of the tumor without reoccurrence [48].

Although AuNPs have a two distinctive absorption bands within the visible region, one on the short axis (transverse) and long axis (longitudinal) [57], enabling their ease of detection, this does cause them to exhibit low quantum yields [58]. So research has inclined towards engineering such NPs into nanocubes in order to heighten quantum yields and so allow for biological tissue detection [58][59]. Furthermore, AuNPs can not only attenuate X-rays for molecular imaging [60], but can be also used in photoacoustic imaging via thermo-elastic expansion, which can be distinguished or identified by ultrasound detectors [61][62].

\section{Porphyrin-Based Magnetic Nanoparticles}

Magnetic nanoparticles are ideal contrast agents applied in MRI ${ }^{[63]}$. In addition, their large surface to volume ratio and biocompatibility facilitate conjugation of targeting ligands and therapeutic drugs onto their surfaces [63]. The main limitations of magnetic nanoparticles are intercellular aggregation and low water solubility; however this can be overcome by encapsulation of hydrophilic polymers onto their surfaces ${ }^{[64]}$.

A multifunctional nanosystem known as $\mathrm{Fe}_{3} \mathrm{O}_{4} @ \mathrm{mSiO}_{2}$ (TPPS)-PL was fabricated by Liuet al. (2017) for the in vitro PDT treatment of HepG2 and HeLa cells ${ }^{[65]}$. The $\mathrm{Fe}_{3} \mathrm{O}_{4}$ magnetic NPs were further coated with mesoporous silica and Pullulan (PL) targeting moieties were covalently attached onto the $\mathrm{Fe}_{3} \mathrm{O}_{4} @ \mathrm{mSiO}_{2}$. Tetraphenylporphyrin tetrasulfonic acid hydrate (TPPS) was then loaded into the silica shell of the nanocomposite to form $\mathrm{Fe}_{3} \mathrm{O}_{4} @ m \mathrm{miO}_{2}$ (TPPS)-PL NPs ${ }^{[65]}$. PL is a water-soluble, nonionic polysaccharide ${ }^{[66]}$ which has a low toxicity and immunogenicity $[65]$. PL binds to the asialoglycoprotein receptor [67] which is overexpressed on HepG2 cells with maximum receptor activity[68]. The cellular uptake results from this study showed that the NPs internalized far better in HepG2 cells than Hela cells, due 
to the high asialoglycoprotein receptor overexpression in HepG2 cells, confirming the effective targeting ability of this nanocomposite [65]. Within PDT in vitro experiments with $200 \mu \mathrm{g} \mathrm{mL} \mathrm{m}^{-1} \mathrm{Fe}_{3} \mathrm{O}_{4} @ \mathrm{mSiO}_{2}$ (TPPS)-PL NPs caused 65\% loss of cell viability in HepG2 cells, while $100 \mathrm{~g} \mathrm{~mL}^{-1}$ of the nanocomposite noted only $20 \%$ of cells remained viable, after 20 min of treatment, suggesting the overall efficacy of this treatment for human hepatoma carcinoma [65].

Thus, the high biocompatibility and biodegradability of magnetic NPs are powerful properties within biomedical applications [69]. Although magnetic NPs like iron oxide NPs are being used in MRI due to their high negative contrast, the detection of negative contrast agents in tissues with low signal intensity, such as lungs and blood clots, is challenging, making cancer diagnosis sometimes difficult $[70]$. In this sense, positive contrast NPs such as manganese oxide NPs are ideal candidates for low signal intensity cancerous tissues [71].

\section{Porphyrin-Based Carbon Nanoparticles}

Various nanocarbons including carbon nanotubes (CNTs), fullerene, nanodiamonds, and carbon nanoparticles (CNPs) are potential assets in phototherapeutic applications [72]. Their tunable surface structure, high drug loading capacity and noticeable fluorescence are promising features of nanocarbon NPs and tubes as PS nanocarriers and theranostic contributors to PDT [5].

Various studies have noted that metal and nitrogen co-doped carbon nanospheres could produce porphyrin-like metal coordination centers, with the ability to change redox states and electronic structures to form active sites [73]74]. In this regard, Chen et al. (2018) synthesized gadolinium encapsulated graphene CNPs (Gd@GCNs) that this synthesized nanoplatform provided a strong spin-orbit coupling at the heavy metal center resulting in significant intersystem crossing and ROS generation under photoirradiation [75]. The mechanism of action of this synthesized nanoplatform has also been proposed in other studies investigating the doping of metal into the core of CNPs [76][77][78]. The synthesized Gd@GCNs showed high singlet oxygen quantum yields and strong fluorescence when applied in dual-modal fluorescence imaging and MRI [75]. This nanocomposite also demonstrated satisfactory image-guided PDT outcomes for the in vivo cancer treatment of SCC-7 tumor induced mouse models. Furthermore, the tumor growth significantly delayed in the mice injected with Gd@GCNs. A tumor inhibition rate of $2.4 \pm 1.6$ was also observed in the PDT group on day 12 (Figure 3) [75].

A

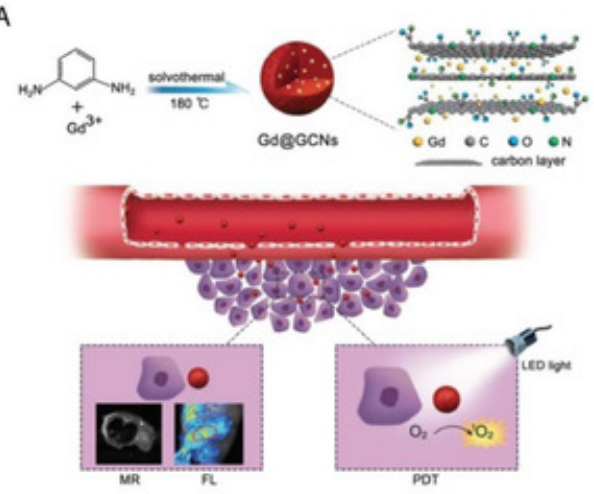

B

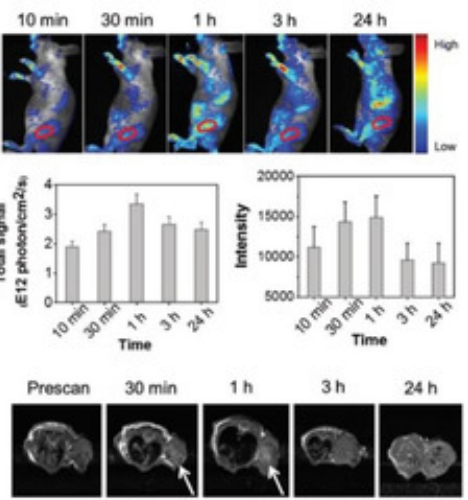

Figure 3. (A) Preparation of Gd@GCNs nanoplatform for imaging-guided phototherapy in solid tumors, B) In vivo fluorescence images acquired from tumor-bearing nude mice after intravenous injection of Gd@GCNs at different time intervals, noting significant tumor shrinkage over time after PDT treatment. Reprinted with permission from ref. [75]. Copyright 2018 Wiley Online Library.

The high surface area and loading capacity, together with the release of vibrational energy induced by NIR exposure are ideal properties CNTs present for theranostic applications [79][80]. However, the low solubility and toxicity of CNTs still require ongoing investigations within clinical tests [81].

\section{Porphyrin-Based Silica Nanoparticles}


The main distinguished features of silica NPs is their distinct nanostructure and well-established siloxane chemistry, which facilitates the use of various methods of surface modifications for diagnostic and therapeutic applications [82][83]. They also enable the fabrication of different silica nanoplatforms with various morphologies, sizes and porosity such as hollow or mesoporous silica NPs [84].

Martínez-Carmona et al. (2017) proposed a visible light-responsive nano-drug delivery which comprised of silica NPs (MSN) decorated with porphyrin-caps to deliver topotecan (TOP) [85]. The authors noted higher tissue penetration of visible light when compared to UV light for the PDT treatment of in vitro HOS osteosarcoma cancer cells. In the presence of visible light, the porphyrin-nanocaps produce singlet oxygen molecules which broke the sensitive-linker and triggered pore uncapping, allowing the release of the entrapped TOP [85]. This nano-drug system was non-toxic and the greater penetration capacity of visible radiation noted a double antitumor effect due TOP release and porphyrin ROS production [85]. Furthermore, in vitro assays revealed that TOP was released in controlled fashion inside HOS osteosarcoma cancer cells, via drug endosome escape to reach the cytoplasm [85][86]. This research work opened up promising expectations for new alternative drug delivery systems for cancer treatment $[85]$.

Chemotherapeutic drugs such as gemcitabine hydrochloride are noted to have a shortin vivo half-life and poor membrane permeability due to its hydrophilic nature [87]. Thus, high amounts of this drug is required to be administered to patients in order to effectively eradicate cancer tumor cells, however this induces adverse unwanted side effects $[87$. In this sense, a nanodelivery system was proposed by Aggad and co-workers (2018) for gemcitabine cancer therapy in order to overcome the hydrophilic limitations of chemotherapeutic drugs [87]. In this study, they synthesized ethylenebased periodic mesoporous organosilica NPs (PMOs) for 2P-PDT and in order to enhance the delivery of gemcitabine within in vitro cultured MCF-7 breast cancer cells [87]. A tetrasilylated porphyrin (PS1) PS was then attached to the ethylene-based PMOs, which caused J-aggregation inside the meso-structure of NPs leading to a two-photon PDT effect [87]. Generally, PSs aggregate with absorption bands shifted to a longer wavelength, than when compared to monomer bands known as J-aggregates, which enhance the two-photon absorption properties [88][89]. The synergistic effect of the two-photon irradiation with gemcitabine delivery and PS1-EPMOs noted more significant cancer cell death than when compared to control cells which did not receive irradiation [87].

Ultra-small hollow silica nanocarriers (HSdots) $(\sim 10 \mathrm{~nm})$ were fabricated within studies performed by Damet al. (2019) [90]. These HSdots were then coated with a porphyrin zinc phthalocyanine (ZnPC) PS for thein vitro PDT treatment of human cutaneous and head/neck squamous cell carcinomas (SCC) cell lines-A431, SCC12 and CAL27, with normal human epidermal keratinocytes (NHEK) as a normal control cells line [90]. Studies have reported that silica nanomaterials are highly biodegradable and so extremely suitable for drug delivery in cancer cells [91]. A higher cellular uptake of ZnPC-loaded HSdots was reported in SCC cell lines, due its cancer targeted modification with folic acid, in comparison to NHEK cells (Figure 4A-D) [90]. Furthermore, the in vitro PDT treatment of SCC cells at $671 \mathrm{~nm}$ laser exposure resulted in more than $90 \%$ cell death, whereas no evidence of dead cells was found in NHEK cells (Figure 4E), suggesting this form of treatment for skin cancer is highly probable[ $\underline{90]}$. 


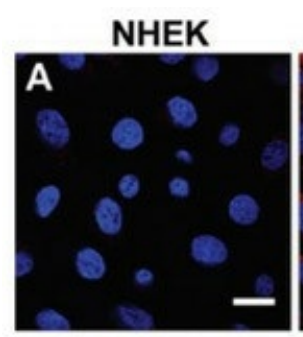

\section{A431}

$\operatorname{scc12}$

CAL27
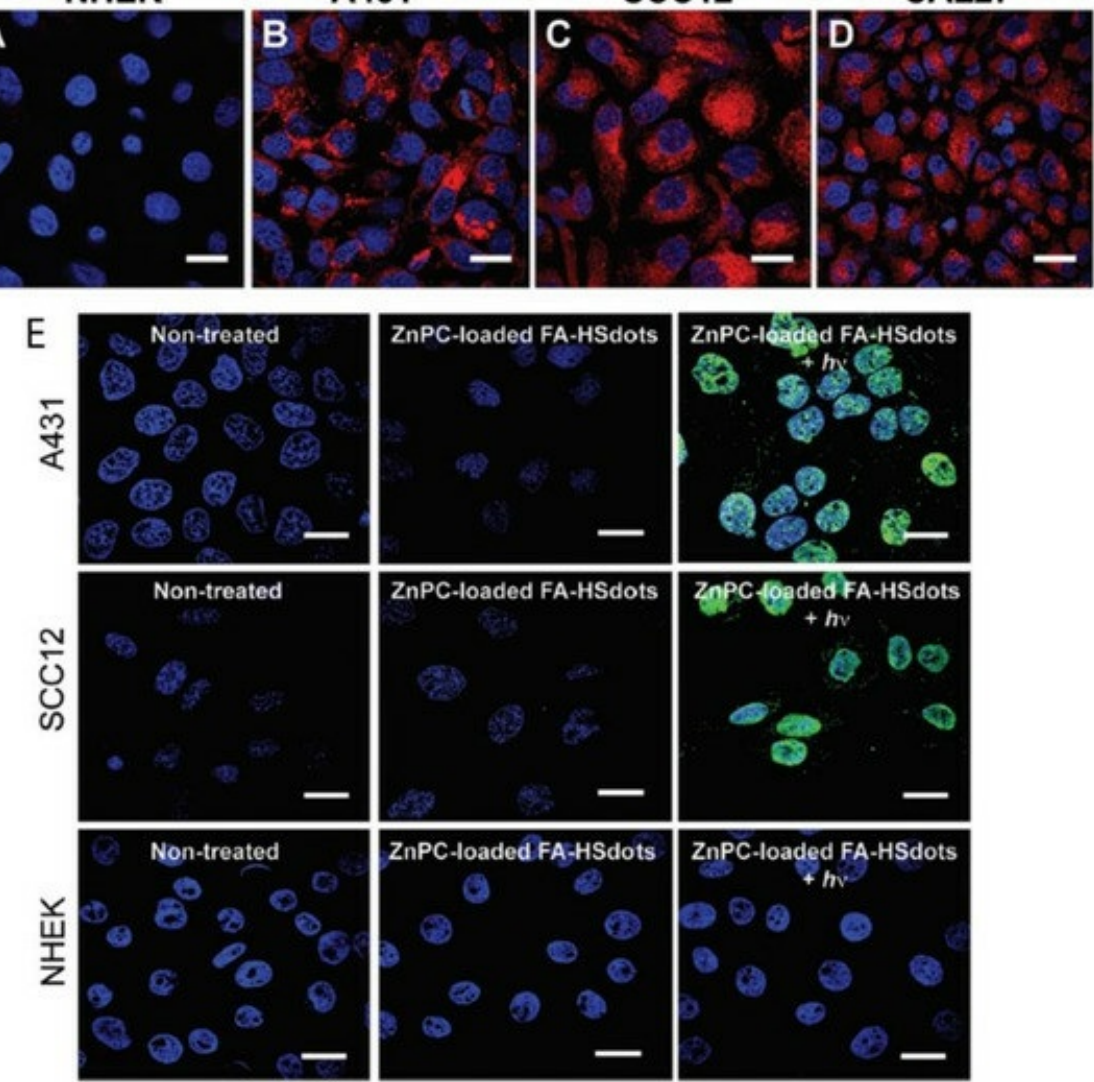

Figure 4. Cellular uptake of ZnPC-loaded FA-HSdots labeled with TRITC in A) normal human epidermal keratinocytes (NHEK) and human cutaneous and head/neck (SCC) lines (B) A431, (C) SCC12, (D) CAL27 and (E) indicates the PDT therapeutic efficacy of ZnPC-loaded FA-HSdots observed in all SCC cells and control NHEK cells after treatment with 2 min laser exposure. Reprinted with permission from ref. [90]. Copyright 2019 Royal Society of Chemistry.

In order to increase the lifetime of nanocarriers in the bloodstream and to delay opsonization and phagocytosis of the NPs, they are often grafted into a protective hydrophilic layer of polysaccharides, such as xylan or long chain polymers of PEG [92[93]. According to studies by Bretinet al. (2019), silica nanoparticles (SNPs) were successfully coated with xylan for the enhanced drug delivery of a 5-(4-hydroxyphenyl)-10,15,20-triphenylporphyrin (TPPOH) PS for the in vitro PDT treatment of human colorectal cancer cells (CRC) ${ }^{[94]}$. Results showed that vectorizing xylan coated SNPs with TPPOH (TPPOH-X SNPs) not only improved anticancer efficacy of PSs PDT in vitro CRC induced apoptotic cell death, also enhanced the in vivo tumor cytotoxicity in CRC xenografted mouse models. In vivo treated mice showed increased apoptosis and cell proliferation inhibition [94].

Multi-modal synergistic phototherapeutic functionalities with NPs have recently been explored by Liet al. (2020) for the treatment of human breast carcinoma (MCF-7) and human lung cancer (A549) in vitro cultured cell lines [95]. Within this study researchers integrated silica nanoparticles (SiNPs) with 5,10,15,20-tetrakis (1-methyl 4-pyridinio) porphyrin tetra (p-toluenesulfonate) (TMPyP) PS and the anticancer drug known as doxorubicin (DOX), to generate singlet oxygen for effective PDT under light irradiation, with chemotherapeutic benefits, respectively [95]. Additionally, due to the two-photon excitation fluorescence abilities of SiNPs, researchers could produce cellular imaging at the NIR laser excitation [95]. Furthermore, Folic acid (FA) was also conjugated to the nanocomposite (MSN@SiNPs@TMPyP-FA) for enhanced targeting capabilities of cancer cells (Figure 5) ${ }^{255}$. Significant blue two-photon fluorescence of the SiNPs from MSN@SiNPs@TMPyP-FA was observed within the cytoplasm of MCF-7 cells, which have more overexpressed FA receptors, in comparison to A549 cells which noted a weaker fluorescence signal, due to their lowered expression

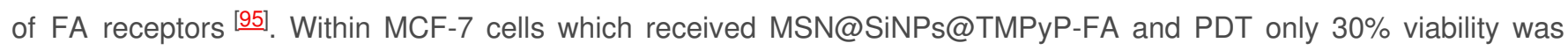
noted, when compared to monotherapy controls [95]. 


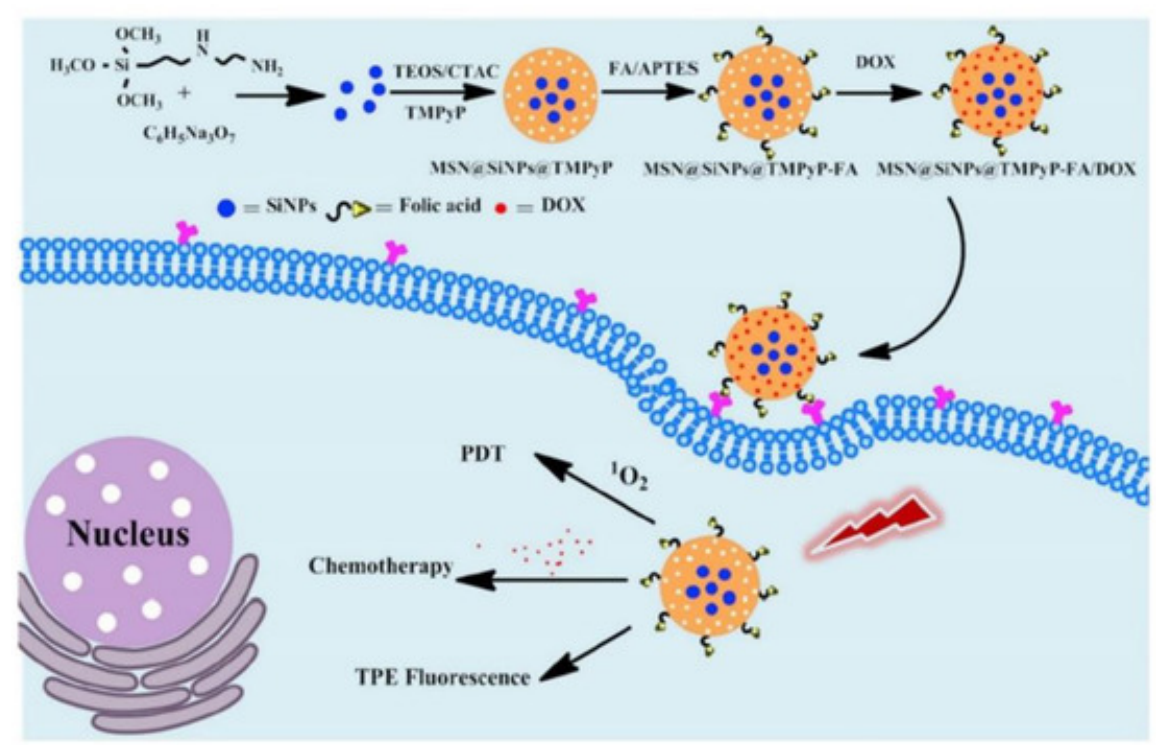

Figure 5. Schematic illustration of the preparation of MSN@SiNPs@TMPyP-FA for enhanced PS and doxorubicin (DOX) drug delivery for improved PDT and chemotherapeutic outcome treatments in cancer cells, with TPE fluorescence. Reprinted with permission from ref. ${ }^{295]}$. Copyright 2020 Elsevier.

Within an in vivo treatment study performed on 4T1 murine breast tumor-bearing mice by Goelet al. (2018), various breast cancer diagnosis and phototherapies such as; PDT-660 nm/PTT-980 nm treatments, positron emission tomography $(\mathrm{PET})$, fluorescence $(\mathrm{FL})$, cerenkov luminescence $(\mathrm{CL})$, and cerenkov radiation energy transfer-based imaging (CRET) were integrated [96]. This study fabricated a multifunctional core-satellite nanoconstruct (CSNC), by coordinating abundant copper sulfide NPs on the surface of hollow mesoporous silica nanoshells (HMSN) decorated with radiolabel $\left.{ }^{89} \mathrm{Zr}\right]{ }^{[96]}$. Meso-tetrakis (4-carboxyphenyl) porphyrin (TCPP) was then conjugated onto the nanohybrid to form a highly efficient multimodal nanotheranostic cancer diagnostic and treatment drug [96]. Overall this multimodal therapy is revolutionary, since it demonstrated the foresight of diagnosis, reported no toxicity and show a significant volume reduction of murine breast tumors without reoccurrence after 30 days [96].

\section{Porphyrin-Based Upconversion Nanoparticles}

Rare earth-based upconversion nanoparticles (UCNPs) have emerged recently in research as a way to circumvent the low tissue penetration depth limitation of PSs [97][98]. Generally, the conversion of NIR light to a shorter wavelength of light is known as upconversion, which is an anti-Stokes process [99]100]. Upconversion luminescence is a nonlinear process whereby successive lower energy photons absorb luminescence and so emit higher energy photons [99][101]. This process has the advantage of low light scattering and autofluorescence background, as well as high tissue penetration depth, since this excitation occurs in the NIR region which is located within phototherapeutic window. This also consequently decreases any photo damage biological tissues might experience ${ }^{[102]}$.

In a study by Sun et al. (2019), protoporphyrin IX PS was modified with jeffamine (PJ) to improve its hydrophilicity and biocompatibility [103]. PEGylated UCNPs were then covalently conjugated to protoporphyrin IX-PJ to control the resonance energy transfer from the UCNPs to the PJs [103]. The authors proposed that the UCNPs-PJ had a tumor targeting ability, due to the presence of low density lipoprotein (LDL) receptors on the surfaces of cancer cells, which enables the passive capture protoporphyrin IX-PJ-UCNPs [103]. Therefore, the study assessed the PDT treatment abilities of this UCNP composite at $980 \mathrm{~nm}$ laser irradiation on an in vitro cultured intestinal human colon adenocarcinoma cell line (LS180) cell line, which is known to over-express LDL receptors [103]. Results reported improved uptake of the PS when conjugated onto the UCNP composite, with significant eradication of cancer cells [103].

A novel caspase-3 responsive functionalized UCNP was fabricated for multi-functional modalities of NIR triggered PDT damage, caspase-3 activation, caspase-3 responsive drug release, and cascade chemotherapeutic activation of DOX by Zhao et al. (2017) [104]. The synthesized UCNPs comprised of pyropheophorbide-a methyl ester (MPPa) PS, tumortargeting CRGD-PEG-DSPE, and caspase-3 responsive DOX prodrug tethered with DEVD peptide (DEVD-DOX) 
forming MPPa/UCNP-DEVD-DOX/Crgd [104]. Upon NIR irradiation, energy from the UCNPs was transferred to the PS which generated ROS for PDT treatment and simultaneously activated caspase-3 to initiate apoptotic cell death (Figure 6) ${ }^{\text {[104]. }}$. The cleavage of the peptide sequence within DEVD-DOX occurred due to the activated caspase-3 enzyme resulting in DOX release for chemotherapy treatment ${ }^{[104]}$. The in vitro NIR PDT treatment of 4 T1 murine breast cancer cells with this multi-functional UCNP demonstrated significantly high cytotoxicity, when compared to control cells which did not receive NIR irradiation [104]. Moreover, in vivo treatment of 4T1 tumor bearing mice with the cascade nanotherapeutic revealed high numbers of pyknotic cells with condensed nuclei, which were either undergoing apoptotic cell death or were completely disintegrated [104].

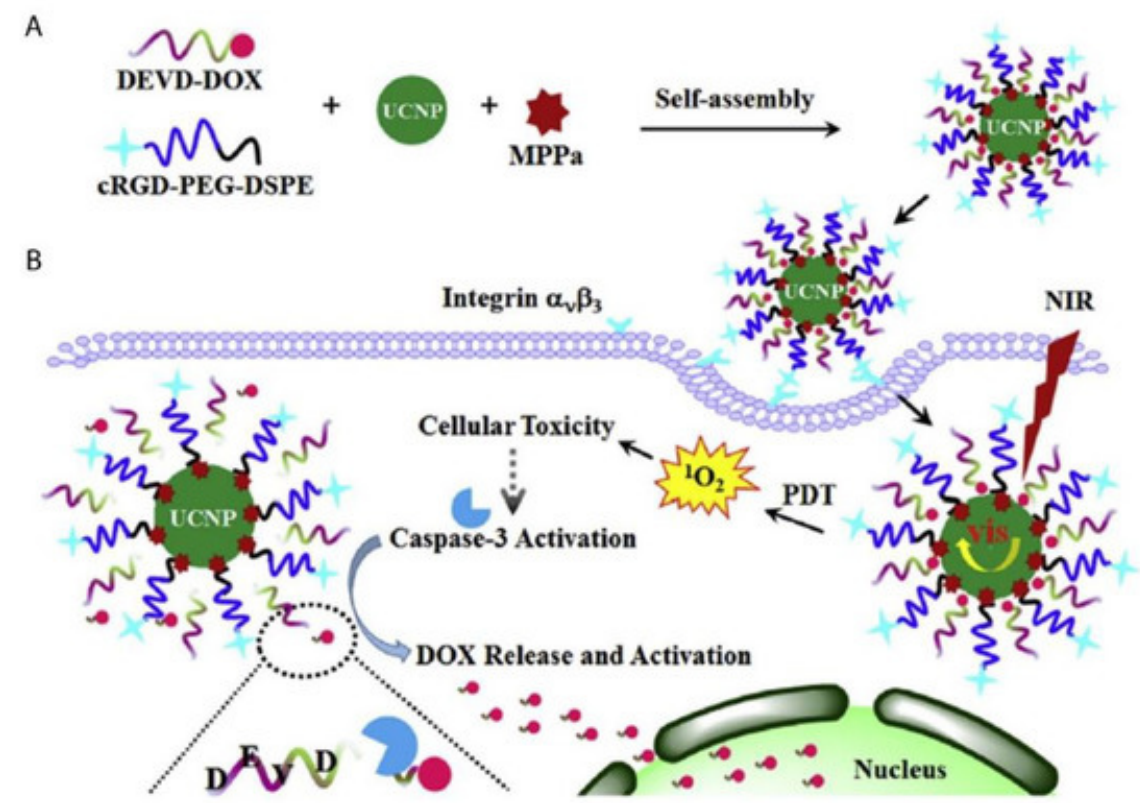

Figure 6. Schematic illustration of (A) fabrication of functionalized upconversion nanoparticles (MPPa/UCNP-DEVDDOX/cRGD), (B) proposed mechanism of PDT action, activation of the caspase-3 and release of chemotherapeutic DOX. Reprinted with permission from ${ }^{[104]}$. Copyright 2017 Elsevier.

Within PDT applied UCNPs, multiple low-energy exciting photons are used to emit a higher energy photon instead of the excitation of a single photon alone [97]. Therefore, UCNPs can achieve improved sensitivity with a low autofluorescence [97]. Furthermore, since UCNP PSs are activated with NIR, the PDT light penetration depth is increased, since NIR can achieve a skin penetration depth of up to $3 \mathrm{~mm}$ [97]. Additionally, UCNP can be excited/activated by $\mathrm{X}$-rays and so are ideal candidates for photodiagnostic detection of deep-seated tumors [71].

\section{Porphyrin-Based Quantum Dots}

Quantum dots (QDs) are small nanocrystals which range from 2 to $10 \mathrm{~nm}$ in size, as well as have unique chemical and physical properties ${ }^{[105]}$. The surface of the QDs can be modified with thiol ligands or amphiphilic copolymers to facilitate bioconjugation of antibodies or small drugs, to improve the solubility and specificity of tumor targeting porphyrin-based PS delivery [105].

Cao et al. (2017) synthesized a porphyrin derivate $(P)$ that reported a high singlet oxygen quantum yield and then conjugated it to PEG and aptamer-functionalized graphene QDs (GQDs), forming multifunctional photodiagnostic and theranostic agent (GQD-PEG-P) for cancer treatment ${ }^{[106]}$. This theranostic nanoplatform was investigated for its PDT/PTT synergic therapy within in vitro cultured A549 lung cancer cells ${ }^{[106]}$. The high fluorescence signal produced by the GQDs allowed for differentiation between A549 lung cancer cells and somatic normal cells and due to their large surface area, it enabled the detection of intracellular cancer-related microRNA (miRNA) in cancer cells [106]. The in vitro combined treatment of A549 cells with PDT/PTT under $635 \mathrm{~nm}$ and $980 \mathrm{~nm}$ laser irradiation could obliterated cancer cells more effectively, when compared to individual treatment modalities administered singularly [106]. Furthermore, MCF-7 breast cancer multicellular tumor spheroids (MCTS) were also assessed for the in vivo GQDPEG-P PDT and PTT phototherapeutic effects and results noted that most cells were dead after treatment ${ }^{106]}$. 
Despite the great properties QDs possess in terms of high tunability with high quantum yield\$71], there are limited studies regarding their application in porphyrin-based PDT cancer treatments. The reason behind the limitation of QD porphyrin-based PDT applications is due to the high toxicity they possess, since most consist of toxic heavy metals like cadmium ions [71]. The application of cadmium free QDs such as zinc and indium-based QDs ${ }^{[107]}$ or the conjugation of QDs to the surface or incorporation into polymeric NPs $[108]$ are a promising area of research, since this enhances their overall application in clinical trials in order to investigate their overall effectiveness for cancer diagnosis and treatment ${ }^{[109]}$. However, due to the limited penetration of visible light, the utilization of both $\mathrm{Cd}$ containing and $\mathrm{Cd}$ free QDs is restrained to superficial tumors for diagnosis of cancer only [71][110]. Nevertheless, QDs possess a high two-photon absorption (TPA) cross-section $\left(\sigma \sim 10^{4} \mathrm{GM}\right)$ [111], which can be employed in two-photon bioimaging ${ }^{[112]}$. Furthermore, polymer-encapsulated QDs (P-QDs) have shown a promising biocompatibility improvement, with minimal cytotoxicity in cells and animals, when compared to standard QDs [112][113].

\section{Conclusions and Perspectives}

Cancer is a dreaded disease causing a vast number of deaths worldwide. Despite ongoing, in-depth research into ways of improving cancer treatment, novel treatment modalities, which obliterate cancer specific tumors, without affecting normal tissues, is still in high demand. The arrival of nanotechnology and engineered nanomedicines have provided a novel opportunity to try and diagnose, as well as treat cancer, by promoting enhanced drug delivery.

Phototherapy is a relatively new unconventional treatment modality that is being investigated for cancer treatment, since it exhibits limited side effects. This review highlights the recent investigations of porphyrin-based nanomedicines for their phototherapeutic and diagnostic applications for cancer over the last 3 years (2017 to 2020). The focus of this review was on the inorganic-based nanoparticles incorporated with porphyrin PSs for phototherapy treatment of cancer.

Various inorganic NPs have been scrutinized for porphyrin delivery. Former porphyrin loaded inorganic NPs in cancer treatment were evaluated by Zhou et al. (2016) ${ }^{[14]}$ (2016) and studies by Xue et al. (2019) thoroughly reviewed porphyrin-based organic NPs for cancer therapy [115]. Therefore, this review serves as a current update from these previous reviews, however with a core focus on inorganic porphyrin-based nanomedicines within PDD, PTT, and PDT application for cancer research.

Porphyrin PSs are the next generation of PSs which can be conjugated to various NP moieties, for improved multiple phototherapy and diagnostic functions in relation to cancer treatment regimes. However, despite its merits, the greatest drawback for porphyrin-based nanomedicines remain the limited penetration of light to deep seated tumors. This remains the greatest challenge when using porphyrin-based nanomedicines for PDT and PTT. Thus, porphyrininorganic NP-based PSs, for now, can only be applied for light accessible tumors, such as skin or bladder cancer [116]. However, recent studies have reported the use of UCNPs, 2P-PDT, and X-rays for cancer phototherapy, where inorganic porphyrin-based NPs can absorb energy at much higher wavelengths, releasing a larger fluorescence signal, as well as allow for a higher wavelength of light which improves penetration of light in tissue and so largely expands their therapeutic and diagnostic applications [117][118][119]. Furthermore, the treatment of cancer metastases is also challenging with porphyrin-based nanomedicines, due to the lack of specific cellular localization. To tackle this limitation, phototherapy using porphyrin-based nanomedicines is often integrated with immunotherapy or targeting ligands and antibodies, which enhance the active uptake of PSs via overexpressed cancer cell receptors [120][121][122]. Lastly, it is important to note that more clinical based in vivo cancer research studies need to be performed in order to completely understand the pharmacological benefits porphyrin-based nanomedicines have ${ }^{[8]}$.

Future directives using inorganic porphyrin NP include the use of PSs in combination with other chemotherapeutic drugs, as well as developing specific targeting nanoplatforms to boost the therapeutic uptake of drugs, limiting offtarget uptake to prevent detrimental side effects and so enhancing cancer treatment.

\section{References}

1. Sasidharan Swarnalatha Lucky; Khee Chee Soo; Yong Zhang; Nanoparticles in photodynamic therapy. Chemical Reviews 2015, 115, 1990-2042, 10.1021/cr5004198.

2. Mark E. Davis; Zhuo (Georgia) Chen and Dong M. Shin. Nanoparticle therapeutics: An emerging treatment modality for cancer. In 
Nanoscience and Technology: A collection of reviews from nature journals; World Scientific: Singapore, Singapore, 2010; pp. 239250.

3. Alyssa Master; Megan Livingston; Anirban Sen Gupta; Photodynamic nanomedicine in the treatment of solid tumors: perspectives and challenges. Journal of Controlled Release 2013, 168, 88-102, 10.1016/j.jconrel.2013.02.020.

4. Ron R Allison; Vanderlei S Bagnato; Claudio H Sibata; Future of oncologic photodynamic therapy. Future Oncology 2010, 6, 929940, 10.2217/fon.10.51.

5. Berihun Sisay; Solomon Abrha; Zewdu Yilma; Admassu Assen; Fantahun Molla; Ebisa Tadese; Abrham Wondimu; Naod GebreSamuel; Gurudutta Pattnaik; Cancer nanotheranostics: a new paradigm of simultaneous diagnosis and therapy. Journal of Drug Delivery and Therapeutics 2014, 4, 79-86, 10.22270/jddt.v4i5.967.

6. H Maeda; J Wu; T Sawa; Y Matsumura; K Hori; Tumor vascular permeability and the EPR effect in macromolecular therapeutics: a review. Journal of Controlled Release 2000, 65, 271-284, 10.1016/s0168-3659(99)00248-5.

7. Fenglin Wang; Chengyao Li; Jing Cheng; Zhiqin Yuan; Recent advances on inorganic nanoparticle-based cancer therapeutic agents. International Journal of Environmental Research and Public Health 2016, 13, 1182, 10.3390/ijerph13121182.

8. Ncediwe Tsolekile; Simphiwe Nelana; Simphiwe Nelana; Porphyrin as diagnostic and therapeutic agent.. Molecules 2019, 24, 2669, 10.3390/molecules24142669.

9. Aisling E. O'Connor; William Gallagher; Annette T. Byrne; Porphyrin and nonporphyrin photosensitizers in oncology: preclinical and clinical advances in photodynamic therapy. Photochemistry and Photobiology 2009, 85, 1053-1074, 10.1111/j.17511097.2009.00585.x.

10. Nela Malatesti; Ivana Munitic; Igor Jurak; Porphyrin-based cationic amphiphilic photosensitisers as potential anticancer, antimicrobial and immunosuppressive agents. Biophysical Reviews 2017, 9, 149-168, 10.1007/s12551-017-0257-7.

11. Shuai Shao; Venugopal Rajendiran; Jonathan F. Lovell; Metalloporphyrin nanoparticles: Coordinating diverse theranostic functions. Coordination Chemistry Reviews 2019, 379, 99-120, 10.1016/j.ccr.2017.09.002.

12. Lawrence P. Cook; Greg Brewer; Winnie Wong-Ng; Structural aspects of porphyrins for functional materials applications. Crystals 2017, 7, 223, 10.3390/cryst7070223.

13. Quanzheng Zha; Xing Rui; Tiantian Wei; Yongshu Xie; Recent advances in the design strategies for porphyrin-based coordination polymers. CrystEngComm 2014, 16, 7371-7384, 10.1039/C4CE00854E.

14. Huijun Phoebe Tham; Hongzhong Chen; Yu Hui Tan; Qiuyu Qu; Sivaramapanicker Sreejith; Lingzhi Zhao; Subbu Venkatraman; Photosensitizer anchored gold nanorods for targeted combinational photothermal and photodynamic therapy. Chemical Communications 2016, 52, 8854-8857, 10.1039/C6CC03076A.

15. Yang Hongying; Wang Fuyuan; Zhang Zhiyi; Photobleaching of chlorins in homogeneous and heterogeneous media. Dyes and Pigments 1999, 43, 109-117, 10.1016/s0143-7208(99)00049-2.

16. Jinfeng Zeng; Wendi Yang; Dongjian Shi; Xiaojie Li; Hongji Zhang; Mingqing Chen; Porphyrin derivative conjugated with gold nanoparticles for dual-modality photodynamic and photothermal therapies In vitro. ACS Biomaterials Science \& Engineering 2018, 4 , 963-972, 10.1021/acsbiomaterials.7b00886.

17. Gonçalo Doria; Joao Conde; Bruno Veigas; L. Giestas; Carina Almeida; Maria Assunção; João Rosa; Pedro V. Baptista; Noble metal nanoparticles for biosensing applications. Sensors 2012, 12, 1657-1687, 10.3390/s120201657.

18. João Conde; Gonçalo Doria; Pedro V. Baptista; Noble metal nanoparticles applications in cancer. Journal of Drug Delivery 2011, 2012, 1-12, 10.1155/2012/751075.

19. Ki Young Choi; Gang Liu; Seulki Lee; Xiaoyuan Chen; Theranostic nanoplatforms for simultaneous cancer imaging and therapy: current approaches and future perspectives. Nanoscale 2012, 4, 330-342, 10.1039/c1nr11277e.

20. Tuan Hiep Tran; Raj Kumar Thapa; Hanh Thuy Nguyen; Tung Thanh Pham; Thiruganesh Ramasamy; Chul Soon Yong; Dong Shik Kim; Jong Oh Kim; Han-Gon Choi; Combined phototherapy in anti-cancer treatment: therapeutics design and perspectives. Journal of Pharmaceutical Investigation 2016, 46, 505-517, 10.1007/s40005-016-0272-x.

21. Oriol Penon; María J. Marín; David Russell; Lluisa Perez-Garcia; Water soluble, multifunctional antibody-porphyrin gold nanoparticles for targeted photodynamic therapy. Journal of Colloid and Interface Science 2017, 496, 100-110, 10.1016/j.jcis.2017.02.006.

22. Qiong Yu; Wei-Xia Xu; Ya-Hong Yao; Zeng-Qi Zhang; Shu Sun; Jun Li; Synthesis and photodynamic activities of a new metronidazole-appended porphyrin and its Zn(II) complex. Journal of Porphyrins and Phthalocyanines 2015, 19, 1107-1113, 10.1142/s1088424615500868.

23. María E. Alea-Reyes; Jorge Soriano; Inmaculada Mora Espí; Ana Mafalda Nunes Rodrigues; David Russell; Leonardo Barrios; Lluisa Perez-Garcia; Amphiphilic gemini pyridinium-mediated incorporation of Zn(II)meso-tetrakis(4-carboxyphenyl)porphyrin into water-soluble gold nanoparticles for photodynamic therapy. Colloids and Surfaces B: Biointerfaces 2017, 158, 602-609, 10.1016/j.colsurfb.2017.07.033.

24. Qingyan Jia; Jiechao Ge; Weimin Liu; Sha Liu; Guangle Niu; Liang Guo; Hongyan Zhang; Pengfei Wang; Gold nanorod@silicacarbon dots as multifunctional phototheranostics for fluorescence and photoacoustic imaging-guided synergistic photodynamic/photothermal therapy. Nanoscale 2016, 8, 13067-13077, 10.1039/c6nr03459d.

25. Ying Wang; Feng Zhang; Qian Wang; Piaoping Yang; Huiming Linab; Fengyu Qu; Hierarchical MoSe2 nanoflowers as novel 
nanocarriers for NIR-light-mediated synergistic photo-thermal/dynamic and chemo-therapy.. Nanoscale 2018, 10, 14534-14545, 10.1039/c8nr04538k.

26. Wen-Xiu Qiu; Li-Han Liu; Shi-Ying Li; Qi Lei; Guo-Feng Luo; Xian-Zheng Zhang; ACPI conjugated gold nanorods as nanoplatform for dual image guided activatable photodynamic and photothermal combined therapy In vivo. Small 2017, 13, 1603956, 10.1002/smll.201603956.

27. Shen Zhang; Hongying Lv; Jing Zhao; Meng Cheng; Shuqing Sun; Synthesis of porphyrin-conjugated silica-coated Au nanorods for synergistic photothermal therapy and photodynamic therapy of tumor. Nanotechnology 2019, 30, 265102, 10.1088/13616528/ab0bd1.

28. Gokhan Yilmaz; Bilal Demir; Suna Timur; C. Remzi Becer; Poly(methacrylic acid)-coated Gold nanoparticles: Functional platforms for theranostic applications. Biomacromolecules 2016, 17, 2901-2911, 10.1021/acs.biomac.6b00706.

29. Omkara Swami Muddineti; Balaram Ghosh; Swati Biswas; Current trends in using polymer coated gold nanoparticles for cancer therapy. International Journal of Pharmaceutics 2015, 484, 252-267, 10.1016/j.ijpharm.2015.02.038.

30. Xuan Wei; Hongzhong Chen; Huijun Phoebe Tham; Nan Zhang; Pengyao Xing; Guangcheng Zhang; Yanli Zhao; Combined photodynamic and photothermal therapy using cross-linked polyphosphazene nanospheres decorated with gold nanoparticles. ACS Applied Nano Materials 2018, 1, 3663-3672, 10.1021/acsanm.8b00776.

31. Xuzhe Wang; Jianwei Fu; Minghuan Wang; Yajie Wang; Zhimin Chen; Jianan Zhang; Jiafu Chen; Qun Xu; Facile synthesis of Au nanoparticles supported on polyphosphazene functionalized carbon nanotubes for catalytic reduction of 4-nitrophenol. Journal of Materials Science 2014, 49, 5056-5065, 10.1007/s10853-014-8212-5.

32. Minghuan Wang; Jianwei Fu; Zhonghui Chen; Xuzhe Wang; Qun Xu; In situ growth of gold nanoparticles onto polyphosphazene microspheres with amino-groups for alcohol oxidation in aqueous solutions. Materials Letters 2015, 143, 201-204, 10.1016/j.matlet.2014.12.114.

33. Ying Hu; Lingjie Meng; Lvye Niu; Qinghua Lu; Facile Synthesis of Superparamagnetic Fe304@polyphosphazene@Au Shells for Magnetic Resonance Imaging and Photothermal Therapy. ACS Applied Materials \& Interfaces 2013, 5, 4586-4591, 10.1021/am400843d.

34. Yang Zhou; Huan Ye; Yongbing Chen; Rongying Zhu; Lichen Yin; Photoresponsive drug/gene delivery systems. Biomacromolecules 2018, 19, 1840-1857, 10.1021/acs.biomac.8b00422.

35. Rongying Zhu; Hua He; Yong Liu; Desheng Cao; Jin Yan; Shanzhou Duan; Yongbing Chen; Lichen Yin; Cancer-selective bioreductive chemotherapy mediated by dual hypoxia-responsive nanomedicine upon photodynamic therapy-induced hypoxia aggravation. Biomacromolecules 2019, 20, 2649-2656, 10.1021/acs.biomac.9b00428.

36. Kok Chan Chong; Fang Hu; Bin Liu; AIEgen bioconjugates for specific detection of disease-related protein biomarkers. Materials Chemistry Frontiers 2019, 3, 12-24, 10.1039/c8qm00383a.

37. Wenpei Fan; Peng Huang; Xiaoyuan Chen; Overcoming the Achilles' heel of photodynamic therapy. Chemical Society Reviews 2016, 45, 6488-6519, 10.1039/C6CS00616G.

38. Huanhuan Fan; Guobei Yan; Zilong Zhao; Xiaoxiao Hu; Wenhan Zhang; Hui Liu; Xiaoyi Fu; Ting Fu; Xiao-Bing Zhang; Weihong Tan; et al. A smart photosensitizer-manganese dioxide nanosystem for enhanced photodynamic therapy by reducing glutathione levels in cancer cells.. Angewandte Chemie International Edition 2016, 55, 5477-5482, 10.1002/anie.201510748.

39. Baoxuan Huang; Jia Tian; Dawei Jiang; Yun Gao; Weian Zhang; NIR-activated "OFF/ON" photodynamic therapy by a hybrid nanoplatform with upper critical solution temperature block copolymers and gold nanorods. Biomacromolecules 2019, 20, 3873-3883, 10.1021/acs.biomac.9b00963.

40. Wei Wu; Wouter Driessen; Xiqun Jiang; Oligo(ethylene glycol)-based thermosensitive dendrimers and their tumor accumulation and penetration. Journal of the American Chemical Society 2014, 136, 3145-3155, 10.1021/ja411457r.

41. Hans Clevers; The cancer stem cell: premises, promises and challenges. Nature Medicine 2011, 17, 313-319, 10.1038/nm.2304.

42. Douglas Hanahan; Robert A Weinberg; Hallmarks of cancer: The next generation. Cell 2011, 144, 646-674, 10.1016/j.cell.2011.02.013.

43. Stephanie M. Pyonteck; Leila Akkari; Alberto J. Schuhmacher; Robert L. Bowman; Lisa Sevenich; Daniela F. Quail; Oakley Olson; Marsha L. Quick; Jason T. Huse; Virginia Teijeiro; et al.Manu SettyChristina S. LeslieYoko OeiAlicia PedrazaJianan ZhangCameron BrennanJames C. SuttonEric C. HollandDylan DanielJohanna A. Joyce CSF-1R inhibition alters macrophage polarization and blocks glioma progression. Nature Medicine 2013, 19, 1264-1272, 10.1038/nm.3337.

44. Yuxun Ding; Jinjian Liu; Yumin Zhang; Xue Li; Hanlin Ou; Tangjian Cheng; Lin Ma; Yingli An; Jianfeng Liu; Fan Huang; et al.Yang LiuLinqi Shi A novel strategy based on a ligand-switchable nanoparticle delivery system for deep tumor penetration. Nanoscale Horizons 2019, 4, 658-666, 10.1039/c8nh00415c.

45. Stanley B. Brown; Two photons are better than one. Nature Photonics 2008, 2, 394-395, 10.1038/nphoton.2008.112.

46. Yizhong Shen; Adam Shuhendler; Deju Ye; Jing-Juan Xu; Huayong Chen; Two-photon excitation nanoparticles for photodynamic therapy. Chemical Society Reviews 2016, 45, 6725-6741, 10.1039/c6cs00442c.

47. Shuang Li; Xiaoqin Shen; Qing-Hua Xu; Yong Cao; Gold nanorod enhanced conjugated polymer/photosensitizer composite nanoparticles for simultaneous two-photon excitation fluorescence imaging and photodynamic therapy.. Nanoscale 2019, 11, 19551- 
19560, 10.1039/c9nr05488j.

48. Shuang Liang; Xiaoran Deng; Yun Chang; Chunqiang Sun; Shuai Shao; Zhongxi Xie; Xiao Xiao; Ping'an Ma; Haiyuan Zhang; Ziyong Cheng; et al.Jun Lin Intelligent hollow Pt-CuS Janus architecture for synergistic catalysis-enhanced sonodynamic and photothermal cancer therapy. Nano Letters 2019, 19, 4134-4145, 10.1021/acs.nanolett.9b01595.

49. Jaber Beik; Ziaeddin Abed; Fatemeh S. Ghoreishi; Samira Hosseini-Nami; Saeed Mehrzadi; Ali Shakeri-Zadeh; Seyed Kamran Kamrava; Nanotechnology in hyperthermia cancer therapy: From fundamental principles to advanced applications. Journal of Controlled Release 2016, 235, 205-221, 10.1016/j.jconrel.2016.05.062.

50. Qian Lv; Huan Min; Dong-Ban Duan; Wei Fang; Gui-Ming Pan; Ai-Guo Shen; Qu-Quan Wang; Guangjun Nie; Ji-Ming Hu; Total aqueous synthesis of Au@Cu2- xS core-shell nanoparticles for in vitro and in vivo SERS/PA imaging-guided photothermal cancer therapy. Advanced Healthcare Materials 2018, 8, 1801257, 10.1002/adhm.201801257.

51. Bo Pang; Yongfeng Zhao; Hannah Luehmann; Xuan Yang; Lisa Detering; Meng You; Chao Zhang; Lei Zhang; Zhi-Yuan Li; Qiushi Ren; et al.Yongjian LiuYounan Xia 64Cu-doped PdCu@Au tripods: A multifunctional nanomaterial for positron emission tomography and image-guided photothermal cancer treatment. ACS Nano 2016, 10, 3121-3131, 10.1021/acsnano.5b07968.

52. Kai Yang; Huan Xu; Liang Cheng; Chunyang Sun; Jun Wang; Zhuang Liu; In vitro and In vivo near-infrared photothermal therapy of cancer using polypyrrole organic nanoparticles. Advanced Materials 2012, 24, 5586-5592, 10.1002/adma.201202625.

53. Yijing Liu; Pravin Bhattarai; Zhifei Dai; Xiaoyuan Chen; Photothermal therapy and photoacoustic imaging via nanotheranostics in fighting cancer.. Chemical Society Reviews 2019, 48, 2053-2108, 10.1039/c8cs00618k.

54. Xiaoqing Huang; Shaoheng Tang; Xiaoliang Mu; Yan Dai; Guangxu Chen; Zhi-You Zhou; Fangxiong Ruan; Zhilin Yang; Nan-Feng Zheng; Freestanding palladium nanosheets with plasmonic and catalytic properties. Nature Nanotechnology 2010, 6, 28-32, 10.1038/nnano.2010.235.

55. Yun Chang; Yan Cheng; Yanlin Feng; Hui Jian; Li Wang; Xiaomin Ma; Xi Li; Haiyuan Zhang; Resonance energy transfer-promoted photothermal and photodynamic performance of gold-copper sulfide yolk-shell nanoparticles for chemophototherapy of cancer. Nano Letters 2018, 18, 886-897, 10.1021/acs.nanolett.7b04162.

56. Santana Bala Lakshmanan; Xiaoju Zou; Marius Hossu; Lun Ma; Chang Yang; Wei Chen; Local field enhanced Au/CuS nanocomposites as efficient photothermal transducer agents for cancer treatment.. Journal of Biomedical Nanotechnology 2012, 8, 883-890, 10.1166/jbn.2012.1486.

57. Nthabeleng Hlapisi; Tshwafo E Motaung; Linda Z. Linganiso; Oluwatobi S. Oluwafemi; Sandile P. Songca; Encapsulation of gold nanorods with porphyrins for the potential treatment of cancer and bacterial diseases: A critical review.. Bioinorganic Chemistry and Applications 2019, 2019, 27, 10.1155/2019/7147128.

58. Xi Wu; Tian Ming; Xin Wang; Peinan Wang; Jianfang Wang; Jiyao Chen; High-photoluminescence-yield gold nanocubes: For cell imaging and photothermal therapy. ACS Nano 2009, 4, 113-120, 10.1021/nn901064m.

59. Haifeng Wang; Terry B. Huff; Daniel A. Zweifel; Wei He; Philip S. Low; Alexander Wei; Ji-Xin Cheng; In vitro and in vivo two-photon luminescence imaging of single gold nanorods. Proceedings of the National Academy of Sciences 2005, 102, 15752-15756, 10.1073/pnas.0504892102.

60. Elodie Boisselier; Didier Astruc; Gold nanoparticles in nanomedicine: preparations, imaging, diagnostics, therapies and toxicity. Chemical Society Reviews 2009, 38, 1759-1782, 10.1039/b806051g.

61. Jelena Levi; Sri Rajasekhar Kothapalli; Te-Jen Ma; Keith Hartman; Butrus T. Khuri-Yakub; Sanjiv Sam Gambhir; Design, synthesis, and imaging of an activatable photoacoustic probe. Journal of the American Chemical Society 2010, 132, 11264-11269, 10.1021/ja104000a.

62. Dipanjan Pan; Manojit Pramanik; Angana Senpan; Soumojit Ghosh; Samuel A. Wickline; Lihong V. Wang; Gregory M. Lanza; Near infrared photoacoustic detection of sentinel lymph nodes with gold nanobeacons. Biomaterials 2010, 31, 4088-4093, 10.1016/j.biomaterials.2010.01.136.

63. Jin Xie; Gang Liu; Henry S. Eden; Hua Ai; Xiaoyuan Chen; Surface-engineered magnetic nanoparticle platforms for cancer imaging and therapy. Accounts of Chemical Research 2011, 44, 883-892, 10.1021/ar200044b.

64. Santimukul Santra; Charalambos Kaittanis; Jan Grimm; J. Manuel Perez; Drug/dye-loaded, multifunctional iron oxide nanoparticles for combined targeted cancer therapy and dual optical/magnetic resonance imaging. Small 2009, 5, 1862-1868, 10.1002/smll.200900389.

65. Xinmeng Liu; Hongli Zhao; Wenxiu Gong; Zhengcheng Zhang; Minbo Lana; Xueling Zhao; Pullulan-functionalized Fe3O4 nanoparticles with mesopore silica-loaded tetraphenylporphyrin tetrasulfonic acid hydrate for targeting photodynamic therapy. Journal of Nanoscience and Nanotechnology 2017, 17, 3880-3887, 10.1166/jnn.2017.13104.

66. Ana Margarida Gonçalves Carvalho Dias; A. Hussain; A.S. Marcos; A.C.A. Roque; A biotechnological perspective on the application of iron oxide magnetic colloids modified with polysaccharides. Biotechnology Advances 2011, 29, 142-155, 10.1016/j.biotechadv.2010.10.003.

67. Jung-Hyun Park; Eun-Wie Cho; Song Yub Shin; Yun- Jung Lee; Kil Lyong Kim; Detection of the asialoglycoprotein receptor on cell lines of extrahepatic origin. Biochemical and Biophysical Research Communications 1998, 244, 304-311, 10.1006/bbrc.1998.8256.

68. J C Collins; E Paietta; R Green; A G Morell; R J Stockert; Biotin-dependent expression of the asialoglycoprotein receptor in HepG2.. 
Journal of Biological Chemistry 1988, 263, 11280-11283.

69. Conroy Sun; Kim Du; Chen Fang; Narayan Bhattarai; Omid Veiseh; Forrest M. Kievit; Zachary Stephen; Nghoon Lee; Richard G. Ellenbogen; Buddy Ratner; et al.Miqin ZhangForrest Kivit PEG-mediated synthesis of highly dispersive multifunctional superparamagnetic nanoparticles: Their physicochemical properties and function in vivo. ACS Nano 2010, 4, 2402-2410, 10.1021/nn100190v.

70. Taeho Kim; Eric Momin; Jonghoon Choi; Kristy Yuan; Hasan Zaidi; Jaeyun Kim; Mihyun Park; Junyoung Lee; Michael T. McMahon; Alfredo Quiñones-Hinojosa; et al.Jeff W. M. BulteTaeghwan HyeonAssaf A. Gilad Mesoporous silica-coated hollow manganese oxide nanoparticles as positive T1 contrast agents for labeling and MRI tracking of adipose-derived mesenchymal stem cells. Journal of the American Chemical Society 2011, 133, 2955-2961, 10.1021/ja1084095.

71. Forrest M. Kievit; Miqin Zhang; Cancer nanotheranostics: Improving imaging and therapy by targeted delivery across biological barriers. Advanced Materials 2011, 23, H217-H247, 10.1002/adma.201102313.

72. Inderbir Singh; Ashish K. Rehni; Pradeep Kumar; Manoj Kumar; Hassan Y. Aboul-Enein; Carbon nanotubes: Synthesis, properties and pharmaceutical applications. Fullerenes, Nanotubes and Carbon Nanostructures 2009, 17, 361-377, 10.1080/15363830903008018.

73. Nghui Guo; Riku Shibuya; Chisato Akiba; Shunsuke Saji; Takahiro Kondo; Junji Nakamura; Active sites of nitrogen-doped carbon materials for oxygen reduction reaction clarified using model catalysts. Science 2016, 351, 361-365, 10.1126/science.aad0832.

74. Shunhao Wang; Lu Shang; Linlin Li; Yingjie Yu; Chongwei Chi; Kun Wang; Jie Zhang; Run Shi; Heyun Shen; Geoffrey I. N. Waterhouse; et al.Sijin LiuJie TianTierui ZhangHuiyu Liu Metal-organic-framework-derived mesoporous carbon nanospheres containing porphyrin-like metal centers for conformal phototherapy. Advanced Materials 2016, 28, 8379-8387, 10.1002/adma.201602197.

75. Hongmin Chen; Yuwei Qiu; Dandan Ding; Huirong Lin; Wenjing Sun; Geoffrey D. Wang; Weicheng Huang; Weizhong Zhang; Daye Lee; Gang Liu; et al.Jin XieXiaoyuan Chen Gadolinium-encapsulated graphene carbon nanotheranostics for imaging-guided photodynamic therapy. Advanced Materials 2018, 30, 1802748, 10.1002/adma.201802748.

76. Enguo Ju; Kai Dong; Zhaowei Chen; Zhen Liu; Chaoqun Liu; Yanyan Huang; Zhenzhen Wang; Fang Pu; Jinsong Ren; Xiaogang Qu; et al. Copper(II)-graphitic carbon nitride triggered synergy: Improved ROS generation and reduced glutathione levels for enhanced photodynamic therapy. Angewandte Chemie 2016, 128, 11639-11643, 10.1002/ange.201605509.

77. Yongxia Zhang; Kadir Aslan; Michael J. R. Previte; Chris D. Geddes; Metal-enhanced singlet oxygen generation: A consequence of plasmon enhanced triplet yields. Journal of Fluorescence 2007, 17, 345-349, 10.1007/s10895-007-0196-y.

78. Wenting Wu; Liying Zhan; Weiyu Fan; Jizhong Song; XiaoMing Li; Zhongtao Li; Ruiqin Wang; Jinqiang Zhang; Jingtang Zheng; Mingbo Wu; et al.Haibo Zeng Cu-N dopants boost electron transfer and photooxidation reactions of carbon dots. Angewandte Chemie International Edition 2015, 54, 6540-6544, 10.1002/anie.201501912.

79. Dimitrios Tasis; Nikos Tagmatarchis; Alberto Bianco; Maurizio Prato; Chemistry of carbon nanotubes. Chemical Reviews 2006, 106, 1105-1136, 10.1021/cr050569o.

80. Andrew Burke; Xuanfeng Ding; Ravi Singh; Robert A. Kraft; Nicole Levi-Polyachenko; Marissa Nichole Rylander; Chris Szot; Cara Buchanan; Jon Whitney; Jessica Fisher; et al.Heather C. HatcherRalph D'agostinoNancy D. KockP. M. AjayanDavid L. CarrollSteven AkmanFrank M. TortiSuzy V. Torti Long-term survival following a single treatment of kidney tumors with multiwalled carbon nanotubes and near-infrared radiation. Proceedings of the National Academy of Sciences 2009, 106, 12897-12902, 10.1073/pnas.0905195106.

81. J.-P. Kaiser; M. Roesslein; T. Buerki-Thurnherr; P. Wick; Carbon nanotubes - Curse or blessing. Current Medicinal Chemistry 2011, 18, 2115-2128, 10.2174/092986711795656171.

82. Li-Sheng Wang; Min-Chieh Chuang; Ja-an Annie Ho; Nanotheranostics - a review of recent publications. International Journal of Nanomedicine 2012, 7, 4679-4695, 10.2147/IJN.S33065.

83. Vahid Shirshahi; Madjid Soltani; Solid silica nanoparticles: applications in molecular imaging. Contrast Media \& Molecular Imaging 2015, 10, 1-17, 10.1002/cmmi.1611.

84. Dalong Ni; Dawei Jiang; Emily B. Ehlerding; Peng Huang; Weibo Cai; Radiolabeling silica-based nanoparticles via coordination chemistry: Basic principles, strategies, and applications. Accounts of Chemical Research 2018, 51, 778-788, 10.1021/acs.accounts.7b00635.

85. Marina Martínez Carmona; R M Lozano; Alejandro Baeza; M. Colilla; María Vallet-Regí; A novel visible light responsive nanosystem for cancer treatment. Nanoscale 2017, 9, 15967-15973, 10.1039/C7NR05050J.

86. Anna M. Sauer; Axel Schlossbauer; Nadia Ruthardt; Valentina Cauda; Thomas Bein; Christoph Bräuchle; Role of endosomal escape for disulfide-based drug delivery from colloidal mesoporous silica evaluated by live-cell imaging. Nano Letters 2010, 10, 3684-3691, $10.1021 / \mathrm{nl} 102180$ s.

87. Dina Aggad; Chiara Mauriello Jimenez; Soraya Dib; Jonas G. Croissant; Laure Lichon; Danielle Laurencin; Sébastien Richeter; Marie Maynadier; Shahad K. Alsaiari; Makhlouf Boufatit; et al.Laurence RaehmMarcel GarciaNiveen M. KhashabMagali Gary-BoboJeanOlivier Durand Gemcitabine delivery and photodynamic therapy in cancer cells via porphyrin-ethylene-based periodic mesoporous organosilica nanoparticles. ChemNanoMat 2018, 4, 46-51, 10.1002/cnma.201700264. 
88. Frank Würthner; Theo E. Kaiser; Chantu R. Saha-Möller; J-aggregates: From serendipitous discovery to supramolecular engineering of functional dye materials. Angewandte Chemie International Edition 2011, 50, 3376-3410, 10.1002/anie.201002307.

89. Sanchita Biswas; Hyo-Yang Ahn; Mykhailo V. Bondar; Kevin D. Belfield; Two-photon absorption enhancement of polymer-templated porphyrin-based J-aggregates. Langmuir 2012, 28, 1515-1522, 10.1021/la203883k.

90. Duncan Hieu M. Dam; Lingzhi Zhao; Sophia A. Jelsma; Yanli Zhao; Amy S. Paller; Folic acid functionalized hollow nanoparticles for selective photodynamic therapy of cutaneous squamous cell carcinoma. Materials Chemistry Frontiers 2019, 3, 1113-1122, 10.1039/c9qm00144a.

91. Dengke Shen; Jianping Yang; Xiaomin Li; Lei Zhou; Renyuan Zhang; Wei Li; Lei Chen; Rui Wang; Fan Zhang; Dongyuan Zhao; et al. Biphase stratification approach to three-dimensional dendritic biodegradable mesoporous silica nanospheres. Nano Letters 2014, 14, 923-932, 10.1021/nl404316v.

92. Caroline LeMarchand; Ruxandra Gref; Patrick Couvreur; Couvreur Patrick; Polysaccharide-decorated nanoparticles. European Journal of Pharmaceutics and Biopharmaceutics 2004, 58, 327-341, 10.1016/j.ejpb.2004.02.016.

93. D Owensiii; Nicholas A. Peppas; Opsonization, biodistribution, and pharmacokinetics of polymeric nanoparticles. International Journal of Pharmaceutics 2006, 307, 93-102, 10.1016/j.ijpharm.2005.10.010.

94. Ludovic Bretin; Aline Pinon; Soukaina Bouramtane; Catherine Ouk; Laurence Richard; Marie-Laure Perrin; Alain Chaunavel; Claire Carrion; Frédérique Brégier; Vincent Sol; et al.Vincent ChaleixDavid Yannick LegerBertrand Liagre Photodynamic therapy activity of new porphyrin-xylan-coated silica Nanoparticles in human colorectal cancer.. Cancers 2019, 11, 1474, 10.3390/cancers11101474.

95. Si Li; Yue Zhang; Xi- Wen He; Wen-You Li; Yu- Kui Zhang; Multifunctional mesoporous silica nanoplatform based on silicon nanoparticles for targeted two-photon-excited fluorescence imaging-guided chemo/photodynamic synergetic therapy in vitro. Talanta 2020, 209, 120552, 10.1016/j.talanta.2019.120552.

96. Shreya Goel; Carolina A. Ferreira; Feng Chen; Paul A. Ellison; Cerise M. Siamof; Todd E Barnhart; Weibo Cai; Todd E. Barnhar; Activatable hybrid nanotheranostics for tetramodal imaging and synergistic photothermal/photodynamic therapy.. Advanced Materials 2018, 30, 1704367, 10.1002/adma.201704367.

97. Feng Wang; Debapriya Banerjee; Yongsheng Liu; Zhuo Chen; Xiaogang Liu; Upconversion nanoparticles in biological labeling, imaging, and therapy. The Analyst 2010, 135, 1839-1854, 10.1039/c0an00144a.

98. Heike S Mader; Peter Kele; Sayed M Saleh; Otto S. Wolfbeis; Sayed M. Saleh; Upconverting luminescent nanoparticles for use in bioconjugation and bioimaging. Current Opinion in Chemical Biology 2010, 14, 582-596, 10.1016/j.cbpa.2010.08.014.

99. Feng Wang; Xiaogang Liu; Recent advances in the chemistry of lanthanide-doped upconversion nanocrystals. Chemical Society Reviews 2009, 38, 976-989, 10.1039/b809132n.

100. Ling-Dong Sun; Ye-Fu Wang; Chun-Hua Yan; Paradigms and challenges for bioapplication of rare earth upconversion luminescent nanoparticles: Small size and tunable emission/excitation spectra. Accounts of Chemical Research 2014, 47, 1001-1009, 10.1021/ar400218t.

101. Annemarie Nadort; Jiangbo Zhao; Ewa M. Goldys; Lanthanide upconversion luminescence at a nanoscale: fundamentals and optical properties. Nanoscale 2016, 8, 13099-13130, 10.1039/c5nr08477f.

102. Jun Yao; Cheng Huang; Chaohui Liu; Mei Yang; Upconversion luminescence nanomaterials: A versatile platform for imaging, sensing, and therapy. Talanta 2020, 208, 120157, 10.1016/j.talanta.2019.120157.

103. Xiaodan Sun; Peisen Zhang; Yi Hou; Yingying Li; Xiaodan Huang; Zihua Wang; Lihong Jing; Mingyuan Gao; Upconversion luminescence mediated photodynamic therapy through hydrophilically engineered porphyrin. Chemical Engineering and Processing: Process Intensification 2019, 142, 107551, 10.1016/j.cep.2019.107551.

104. Na Zhao; Baoyan Wu; Xianglong Hu; Da Xing; NIR-triggered high-efficient photodynamic and chemo-cascade therapy using caspase-3 responsive functionalized upconversion nanoparticles. Biomaterials 2017, 141, 40-49, 10.1016/j.biomaterials.2017.06.031.

105. Babak Kateb; Katherine Chiu; Keith L. Black; Vicky Yamamoto; Bhavraj Khalsa; Julia Y. Ljubimova; Hui Ding; Rameshwar Patil; Jose Antonio Portilla-Arias; Michel Modo; et al.David F. MooreKeyvan FarahaniMichael S. OkunNeal PrakashJosh NemanDaniel AhdootWarren GrundfestShouleh NikzadJohn D. Heiss Nanoplatforms for constructing new approaches to cancer treatment, imaging, and drug delivery: What should be the policy?. Neuroimage 2011, 54, S106-S124, 10.1016/j.neuroimage.2010.01.105.

106. Yu Cao; Haifeng Dong; Zhou Yang; Xiangmin Zhong; Yi Chen; Wenhao Dai; Xueji Zhang; Aptamer-conjugated graphene quantum dots/porphyrin derivative Theranostic agent for intracellular cancer-related MicroRNA detection and fluorescence-guided photothermal/photodynamic synergetic therapy. ACS Applied Materials \& Interfaces 2017, 9, 159-166, 10.1021/acsami.6b13150.

107. Ken-Tye Yong; Hong Ding; Indrajit Roy; Wing-Cheung Law; Earl J. Bergey; Anirban Maitra; Paras Prasad; Imaging pancreatic cancer using bioconjugated InP quantum dots. ACS Nano 2009, 3, 502-510, 10.1021/nn8008933.

108. Kevin C. Weng; Charles O. Noble; Brigitte Papahadjopoulos-Sternberg; Fanqing F. Chen; Daryl C. Drummond; Dmitri B. Kirpotin; Nghui Wang; Yun K. Hom; Byron Hann; John W. Park; et al. Targeted tumor cell internalization and imaging of multifunctional quantum dot-conjugated immunoliposomes in vitro and in vivo. Nano Letters 2008, 8, 2851-2857, 10.1021/nl801488u.

109. Pavel Zrazhevskiy; Mark Sena; Xiaohu Gao; Designing multifunctional quantum dots for bioimaging, detection, and drug delivery. Chemical Society Reviews 2010, 39, 4326-4354, 10.1039/b915139g. 
110. Min-Kyung So; Chenjie Xu; Andreas Loening; Sanjiv Sam Gambhir; Jianghong Rao; Self-illuminating quantum dot conjugates for in vivo imaging. Nature Biotechnology 2006, 24, 339-343, 10.1038/nbt1188.

111. Daniel R. Larson; Warren R Zipfel; Rebecca M. Williams; Stephen W. Clark; Marcel Bruchez; Frank W. Wise; Watt W. Webb; Watersoluble quantum dots for multiphoton fluorescence imaging in vivo. Science 2003, 300, 1434-1436, 10.1126/science.1083780.

112. Xiaohu Gao; Yuanyuan Cui; Richard M Levenson; Leland W K Chung; Shuming Nie; In vivo cancer targeting and imaging with semiconductor quantum dots. Nature Biotechnology 2004, 22, 969-976, 10.1038/nbt994.

113. Yanyan Fan; Helin Liu; Rongcheng Han; Lu Huang; Hao Shi; Yinlin Sha; Yuqiang Jiang; Extremely high brightness from polymerencapsulated quantum dots for two-photon cellular and deep-tissue imaging. Scientific Reports 2015, 5, 9908, 10.1038/srep09908.

114. Yiming Zhou; Xiaolong Liang; Zhifei Dai; Porphyrin-loaded nanoparticles for cancer theranostics. Nanoscale 2016, 8, 12394-12405, 10.1039/c5nr07849k.

115. Xiangdong Xue; Aaron Lindstrom; Yuanpei Li; Porphyrin-based nanomedicines for cancer treatment. Bioconjugate Chemistry 2019, 30, 1585-1603, 10.1021/acs.bioconjchem.9b00231.

116. Charles J. Gomer; Nicholas J. Razum; Acute skin response in albino mice following porphyrin photosensitization under oxic and anoxic conditions. Photochemistry and Photobiology 1984, 40, 435-439, 10.1111/j.1751-1097.1984.tb04614.x.

117. Xiaoju Zou; Mingzhen Yao; Lun Ma; Marius Hossu; Xiumei Han; Petras Juzenas; Wei Chen; X-ray-induced nanoparticle-based photodynamic therapy of cancer. Nanomedicine 2014, 9, 2339-2351, 10.2217/nnm.13.198.

118. Samana Shrestha; Jing Wu; Bindeshwar Sah; Adam Vanasse; Leon N Cooper; Lun Ma; Gen Li; Huibin Zheng; Wei Chen; Michael P. Antosh; et al. X-ray induced photodynamic therapy with copper-cysteamine nanoparticles in mice tumors.. Proceedings of the National Academy of Sciences 2019, 116, 16823-16828, 10.1073/pnas.1900502116.

119. Benjamin Cline; Ian Delahunty; Jin Xie; Nanoparticles to mediate X-ray-induced photodynamic therapy and Cherenkov radiation photodynamic therapy. Wiley Interdisciplinary Reviews: Nanomedicine and Nanobiotechnology 2018, 11, e1541, 10.1002/wnan.1541.

120. Wen Song; Jing Kuang; Chu-Xin Li; Mingkang Zhang; Diwei Zheng; Xuan Zeng; Chuanjun Liu; Xian-Zheng Zhang; Enhanced immunotherapy based on photodynamic therapy for both primary and lung metastasis tumor eradication. ACS Nano 2018, 12, 19781989, 10.1021/acsnano.7b09112.

121. Guangxu Lan; KaiYuan Ni; ZiWan Xu; Samuel S. Veroneau; Yang Song; Wenbin Lin; Nanoscale metal-organic framework overcomes hypoxia for photodynamic therapy primed cancer immunotherapy. Journal of the American Chemical Society 2018, 140, 5670-5673, 10.1021/jacs.8b01072.

122. Chunbai He; Xiaopin Duan; Nining Guo; Christina Chan; Christopher Poon; Ralph R. Weichselbaum; Wenbin Lin; Core-shell nanoscale coordination polymers combine chemotherapy and photodynamic therapy to potentiate checkpoint blockade cancer immunotherapy. Nature Communications 2016, 7, 1-12, 10.1038/ncomms12499.

\section{Keywords}

Porphyrins; nanotheranostics; inorganic nanoparticles; cancer treatment

(C) 2020 by the author(s). Distribute under a Creative Commans CC BY license 